\title{
An introduction to mathematical and numerical modeling of heart electrophysiology
}

\author{
Luca Gerardo-Giorda
}

\begin{abstract}
The electrical activation of the heart is the biological process that regulates the contraction of the cardiac muscle, allowing it to pump blood to the whole body. In physiological conditions, the pacemaker cells of the sinoatrial node generate an action potential (a sudden variation of the cell transmembrane potential) which, following preferential conduction pathways, propagates throughout the heart walls and triggers the contraction of the heart chambers.

The action potential propagation can be mathematically described by coupling a model for the ionic currents, flowing through the membrane of a single cell, with a macroscopical model that describes the propagation of the electrical signal in the cardiac tissue. The most accurate model available in the literature for the description of the macroscopic propagation in the muscle is the Bidomain model, a degenerate parabolic system composed of two non-linear partial differential equations for the intracellular and extracellular potential. In this paper, we present an introduction to the fundamental aspects of mathematical modeling and numerical simulation in cardiac electrophysiology.
\end{abstract}

\section{Introduction}

Cardiac-specific diseases account for 700,000 deaths each year in Europe, half of this mortality being due to heart failure (ineffective contraction, principally due to ventricular dyssynchrony). The other half of cardiac mortality occurs suddenly, essentially due to ventricular tachyarrhythmias. Although the vast majority of these cases is associated with chronic cardiac disease, sudden cardiac death can also occur in seemingly healthy and sometimes very young people. Knowledge about the underlying causes and options for diagnosis and prevention is still very limited. Still,

Luca Gerardo-Giorda

BCAM - Basque Center for Applied Mathematics, Alameda Mazarredo 14, 48009 Bilbao, Spain e-mail: lgerardo@bcamath.org 
with 6 million individuals suffering from atrial fibrillation, 9 million people affected by heart failure, and 350,000 sudden deaths every year, the human and economic burden of cardiac electrical diseases skyrocketed in Europe. The estimated annual direct costs for the health care system in Europe is topping 1100 billion Euros.

The contraction of the heart is orchestrated by a complex mechanism of electrical activation. As a consequence, an important part of heart failure occurrencies is caused or aggravated by electrical dysfunctions, and heart electrophysiology has become in the recent years the subject of a vast interdisciplinary literature, from medical sciences through bio-engineering, physiology, chemistry and physics.

The electrical activity of the heart as a whole is characterized by a complex multiscale structure, ranging from the microscopic activity of ion channels in the cellular membrane to the macroscopic properties of the anisotropic propagation of the excitation and recovery fronts in the whole heart. Cardiac arrhythmias are complex disruptions of this organisation.

Cardiac cells, called myocytes, are a particular type of excitable cells. At resting condition, they feature a negative transmembrane potential, resulting from the difference between internal and external concentrations of charged ions $\left([\mathrm{Na}]^{+},[\mathrm{K}]^{+}\right.$, $\left.[\mathrm{Ca}]^{++}\right)$. A small change of the cell's environment from its rest state produces a very fast depolarization, followed by a slower repolarization process towards the resting state. This cellular activity is called Action Potential (AP), and is mathematically described by an ionic ODE model which is the basis of dynamic behaviour in the model. Modern detailed ionic models take into account transmembrane current flows, intracellular calcium handling, and can include energetics and force production $[15,41]$

Higher levels of complexity govern the propagation of the electrical impulse for optimal contraction at the tissue and organ levels. The global activation sequence of the heart follows from the physical organization of a special conduction network that is essential for the synchronization of the whole heart. In healthy conditions, atria and ventricles are electrically insulated from each other and are connected only through the atrioventricular node (AV). The AP originates in the sinoatrial node (SA), propagates in the atria through Bachmann's bundle, it is modulated in frequency by the $\mathrm{AV}$, and proceeds to the ventricles, where the His-Purkinje system (PS) provides a preferencial pathway for the AP to propagate through the lower chambers.

The difficulty in having access to direct measures on real patients fueled the interest in mathematical modeling and numerical simulations, which have been supporting cardiovascular science for more than 20 years. In this respect, cardiac modeling in medicine has significantly evolved in the recent decades, providing the best and highly detailed mathematical description of any organ system in the body. Many fundamental insights have been gained from in-silico experiments [65], even ahead of experimental evidence [55]. Numerical models and fast dedicated solvers already exist and allow in-silico exploration of the mechanisms underlying these pathologies at the cost of large-scale simulations.

If on the one hand, modern imaging techniques, such as high-resolution magnetic resonance imaging (MRI), allows high level of accuracy in the description of 
both the microstructure of the tissue and the global anatomy of the organ, current mathematical models are based on a formalism (the Bidomain equations, [38, 14]) whose derivation is based on a very simplified geometry with respect to the nowadays available structural data. The heart is, in fact, a collection of interconnected excitable tissues, each of which has specific modelling requirements.

Each of these tissues is a complex network of cardiomyocytes connected to each other by gap-junctions, together with other types of cells (fibroblasts) and collagen. APs propagate from cell to cell, resulting in AP waves at the macroscopic tissue scale. From a mathematical standpoint, a multiscale technique allows to model the tissue, at the macroscopic level, as a continuum wher the intra- and extra-cellular media are superimposed and the corresponding potentials are the solutions to a system of degenerate partial differential equation of reaction-diffusion type coupled over space with the system of ODEs (the ionic model). Such model is known as the Bidomain system of equations. In this model, the anisotropies of the intra- and extracellular conductivities differ. In case of an insulated tissue and under the so-called equal anisotropy ratio assumption, the system reduces to a single reaction-diffusion equation: the Monodomain equation. The Monodomain equation is no longer degenerate, thus far cheaper to solve numerically [34].

The numerical approximation of the Bidomain model is often based on a finite element discretization in space and on implicit-explicit time advancing schemes (IMEX): the ionic variables are advanced to the current time step, and inserted in the nonlinear term, while the latter one is then linearized around the value of the membrane potential at the previous time step. The degenerate parabolic nature of the Bidomain system, however, entails a very ill conditioning for the linear system associated to its discretization. From the mathematical and numerical standpoint, many efforts have been devoted in the recent years to set up efficient solvers and preconditioners to reduce the high computational costs associated to its numerical solution [63, 44, 62, 5, 10, 47]. Many proposed preconditioning strategies have been based on multigrid approaches $[45,64,54]$ or suitable approximations of the equations [23, 22]. Among these works, most are based on a proper decomposition of the computational domain in order to set up parallel preconditioners, or on suitable multigrid schemes still coupled with parallel architectures [42, 60]. In particular, a Classical Schwarz Method coupled with a multigrid approach has been proposed in [43], while an Optimized Schwarz method has been introduced in [25]. The stiffness of the problem, due to the presence of a steep propagation front, led to the introduction of adaptive schemes, in both time [46], and time and space [7]. Another approach has been aiming at a simplification of the original problem, by using a somehow optimized Monodomain model [39], and by developing model adaptive techniques, where the costly Bidomain model is replaced by the Monodomain one (or an extended version of it) far from the depolarization front and the recovery tail of the action potential [26, 25, 24]. In the rest of the paper we provide a general survey of the mathematical and numerical aspects of the cardiac electrophysiology modeling. Section 2 is devoted to the modeling of an excitable myocardial cell, and some ionic models are presented: a phenomelogical one, a model for atrial myocytes and a model for ventricular ones. In Section 3 we present a derivation of the 
macroscopic Bidomain model for propagation and its simplified version, the Monodomain. Section 4 provides an introduction to the numerical approximation of both Bidomain and Monodomain models.

\section{Mathematical modeling of an excitable myocardial cell}

The basic property of neural cells to produce signals is called Action Potential (AP). It consists of a sudden variation in the transmembrane potential, called upstroke, followed by a recovering of the resting condition. It shows different shapes and amplitudes according to the different kind of excitable media to which the cells belong to, and in the large muscle cells makes it possible the simultaneous contraction of the whole cell. An action potential propagates keeping the same shape and amplitude all along an entire neural or muscular fiber. The Action Potential propagates across the heart in an heterogeneous way. The pulse moves from the Sinoatrial Nodus (SA), and propagates through the ordinary myocardic fibers of the right atrium, while the Buchmann's bundle drives the pulse towards the left atrium. Some action potentials propagate downwards and reach the Atrioventricular Nodus (AV), which is, under normal conditions, the only gate for the pulse to propagate from atria to ventricles, where the conduction is quicker $\left(4 \mathrm{~ms}^{-1}\right.$ versus $\left.1 \mathrm{~ms}^{-1}\right)$.

Cardiac cells are characterized by a transmembrane potential that is negative at rest, owing to the fact that the concentration of potassium ions $\left[\mathrm{K}^{+}\right]_{i}$ inside the cardiac cell is remarkably higher than the outside concentration $\left[\mathrm{K}^{+}\right]_{e}$, and show two kinds of action potentials: the quick and the slow response.

The quick response is typical in the myocardium fibers (both atrial and ventricular) and in the Purkinje fibers, which are fibers specialized in the conduction. The quick response cells are characterized by a negative transmembrane potential at rest (around $-90 \mathrm{mV}$ ), and by a rapid depolarization (positive overshoot), where the potential difference changes sign and the internal potential overtakes the external one of around 20mV: such phase is called Phase 0. Immediately after that (Phase 1) a short period of partial repolarization takes place, followed by a plateau (Phase 2) which lasts for around 0.2 seconds. The potential gets progressively more negative (Phase 3) until it reaches again the resting value. The repolarization procedure is far slower than the depolarization one, and the interval between the end of the repolarization and the next action potential is called Phase 4.

The slow response is the one taking place in the Sinoatrial Nodus (SA), the natural pacemaker of the heart, and in the Atrioventricular Nodus (AV), the tissue meant to transfer the pulse from atria to ventricles. The slow response cells are characterized by a resting potential less negative (around $-50 \mathrm{mV}$ ), by a smaller slope and amplitude in the overshoot of the action potential, by the absence of the Phase 1, and by a relative refractory period that continues during Phase 4 .

From the modeling standpoint, the electrical activity in myocites is characterised by transmembrane ionic currents and voltage changes, whose temporal dynamics are governed by the presence of various players at the molecular level (ion channels, 
pumps, concentrations), as well as many different proteins (such as transporters) that are spatially organized at the cellular scale to generate action potentials (AP). The cell membrane is modeled as a capacitor separating the intra- and extra-cellular media, two ionic solutions. In the framework of Hodgkin-Huxley (HH) formalism [28], state variables are associated with the membrane potential, ionic concentrations, and molecular actors such as gating variables, which handle opening and closing of ionic channels. The system dynamics is thus described by a set of differential equations which depend on time, voltage, ion concentrations and the gating variables. Some recent models include additional differential equations to describe calcium regulation within the cell and possibly mitochondrial activity or force generation. Ionic models consist generally of 10-50 ODEs ([57]), but if molecular actors are modelled by Markov processes, such number can grow up to 100 ODEs, [29]. These systems are highly non-linear and extremely stiff because of the large range of time-scales necessary to represent the various phenomena involved (from $100 \mathrm{~ms}$ to $1 \mu \mathrm{s}$ ).

The earliest model for AP appeared in the work on nerve action potential by Hodgkin and Huxley ([28]), which earned them the Nobel prize in Medicine in 1963. Models of this type have successively been developed for the cardiac action potential, where the variation in time of the membrane potential $u$ (under the assumption of an equipotential cell) is given by

$$
C_{m} \frac{d u}{d t}=-I_{i o n}(u, w)+I_{s t},
$$

where $I_{i o n}$ and $I_{s t}$ are the total ionic current and stimulus current across the membrane, respectively, and $C_{m}$ is the total membrane capacitance. In (1) the ionic current through the channels in the membrane depends on the transmembrane potential $u$ and on $M$ gating and concentration variables $w \in \mathbf{R}^{M}$ :

$$
I_{i o n}(u, w)=\sum_{k=1}^{N} G_{k}(u) \prod_{j=1}^{M} w_{j}^{p_{j k}}\left(u-E_{k}(w)\right),
$$

$G_{k}(u)$ being the membrane conductance, $E_{k}$ being the reversal potential for the $k$-th current and $p_{j k}$ being integers, and where the dynamics of the gating and concentration variables is described by a system of ODE's

$$
\frac{d w}{d t}=R(u, w), \quad w(\mathbf{x}, 0)=w_{0}(\mathbf{x}) .
$$

In such models, if $w_{j}$ is a gating variable, the right hand side $R_{j}(u, w)$ has a special structure and the corresponding ODE is given by

$$
\frac{d w_{j}}{d t}=R_{j}(u, w)=R_{j}\left(u, w_{j}\right)=\alpha_{j}(u)\left(1-w_{j}\right)-\beta_{j}(u) w_{j},
$$

with $\alpha_{j}(u), \beta_{j}(u)>0,0<w_{j}<1$. Within this formalism, the temporal variation of a gating variable $y$ equals the difference between the opening rate of closed gates and the closing rate of open gates, with rates that are voltage-dependent. If we introduce 
the steady state of the gating variable with the cell at rest, $y^{\infty}$, and the time constant associated with the gating variable $\tau_{y}$, defined as

$$
y^{\infty}=\alpha_{y}(u) \tau_{y}(u) \quad \tau_{y}(u)=\frac{1}{\alpha_{y}(u)+\beta_{y}(u)},
$$

we observe that the generic gating variable $y$ satisfies the equivalent ordinary differential equation

$$
\frac{d y}{d t}=\frac{y^{\infty}-y}{\tau_{y}}
$$

Concerning the modeling of ventricular cells, the fitting of improved experimental data with more complex models led to the developement of many refinements of the original Hodgkin-Huxley model: among them, we recall the model by Beeler and Reuter (1977, with 4 ionic currents and 7 gating and concentrations variables), and the phase-I Luo-Rudy (1991, with $N=6$ and $M=7$ ). In this direction, the most used model of mammalian ventricular cells is the phase-II Luo-Rudy (1994, [35]), which is based on measurements from guinea pig. Simpler models of reduced complexity have also been proposed, where only 1 or 2 gating variables are considered. In the remainder of this section we present, as an example three ionic models: a 2 variables, phenomenological, model, a detailed model for atrial cells, and a detailed model for ventricular cells.

\subsection{The FitzHugh-Nagumo cell model and its Rogers-McCulloch variant}

The simplest ionic model for an excitable cell is the phenomenological FitzHughNagumo (FHN, [20]) model, consisting of 1 ionic current and 1 gating variable. The latter is a simplified version of the Hodgkin-Huxley model. Assuming the potential $v$ to be zero at rest, the ionic current uses only one recovery variable:

$$
I_{i o n}(u, w)=u-\frac{u^{3}}{3}-w+I,
$$

where $I$ is a stimulus current, and $w$ satisfies

$$
\frac{\partial w}{\partial t}=u+a-b w
$$

with $a, b>0$.

If the stimulus current $I$ exceeds a given threshold, the system exhibits a spike and recovery dynamics. In Figure 1 (left) we plot the temporal evolution of the membrane potential $u$ (solid) and the recovery variable $w$ (dashed).

An improvement of this model is given by the variant introduced in [49] by Rogers and McCulloch, where the ionic current and the recovery variable $w$ dy- 

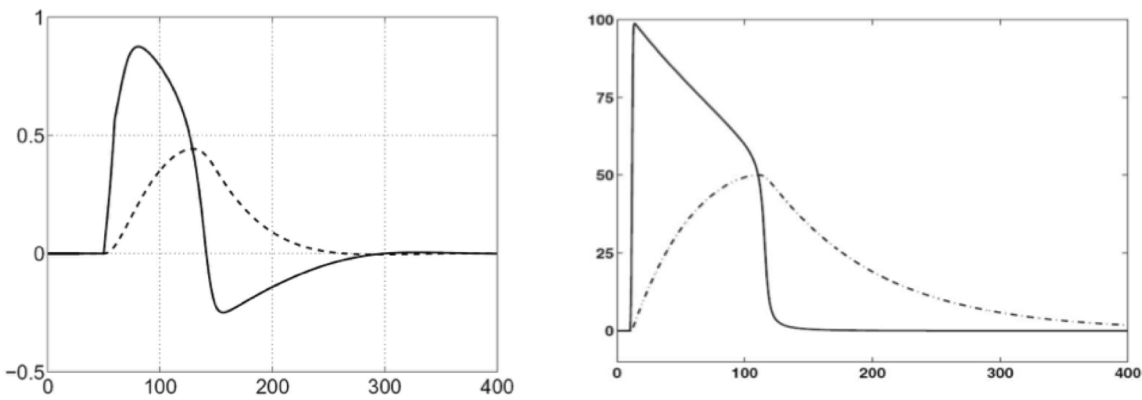

Fig. 1 Time evolution of the membrane potential $u$ (solid line) and the recovery variable $w$ (dashed line) in the FHN model (left) and its Rogers-McCulloch variant (right).

namics are given by

$$
I_{i o n}(u, w)=G u\left(1-\frac{u}{u_{\mathrm{th}}}\right)\left(1-\frac{u}{u_{\mathrm{p}}}\right)+\eta_{1} u w,
$$

and

$$
\frac{\partial w}{\partial t}=\eta_{2}\left(\frac{u}{u_{\mathrm{p}}}-\eta_{3} w\right)
$$

where $G, \eta_{1}, \eta_{2}, \eta_{3}$ are positive coefficients, $u_{\mathrm{th}}$ is a threshold potential, and $u_{\mathrm{p}}$ is the peak potential. If the membrane potential does not exceed the threshold $u_{\mathrm{th}}$, the $\mathrm{AP}$ is not triggered and the system gets back to the resting state. In Figure 1 (right) we report the time evolution of the potential $u$ (solid line) and of the gating variable $w$ (dashed line) for the Rogers-McCulloch variant of the FHN model.

The recovery variable $w$ ensures that, once an AP is triggered, the system cannot be excited again, unless a refractory period has passed. When a stimulus is introduced, the response of the system depends on the elapsed time since its spiking: if the recovery variable is small enough (or, equivalently, enough time has elapsed) another AP is created, with the same shape and amplitude of the first one. If the elapsed time does not outlast the refractory period, the generated AP can be shorter in duration, and smaller in amplitude, or just not being triggered, in the case too little time since spiking has elapsed.

Other all-or-nothing response models have been introduced in the literature, among which we recall the one proposed by Panfilov, Ten-Tusscher, and collaborators in [57]. The great simplicity of such model, and yet its ability in capturing significant aspects of the electrocardiac dynamics, is behind its wide use in literature. However, if on the one hand, such model is well suited to describe the positive overshoot in the quick depolarization phase, on the other hand it provides only a coarse approximation in the plateau and repolarization phases of the action potential, and behaves too poorly when accuracy in the description of the action potential is needed. 


\subsection{The Courtemanche, Ramirez and Nattel atrial cell model}

One of the most accurate models for atrial cells is the one proposed by Courtemanche, Ramirez and Nattel, (CRN, [18]). The total ionic current for the CRN model is given by the sum

$$
I_{\text {ion }}=I_{\mathrm{Na}}+I_{\mathrm{K}}+I_{\mathrm{Ca}}+I_{\mathrm{b}}+I_{\mathrm{p}}
$$

The above expression takes into account several aspects of the action potential generation. In (5), $I_{\mathrm{Na}}$ is the fast depolarizing $\mathrm{Na}^{+}$current, while the quantity $I_{\mathrm{K}}$ is the total rectifier $\mathrm{K}^{+}$current, given by

$$
I_{\mathrm{K}}=I_{\mathrm{K} 1}+I_{\mathrm{to}}+I_{\mathrm{Kur}}+I_{\mathrm{Kr}}+I_{\mathrm{Ks}},
$$

where $I_{\mathrm{K} 1}$ is the inward rectifier $\mathrm{K}^{+}$current, playing a major role in the late repolarization phase of the AP and in determining resting membrane potential and resistance, $I_{\mathrm{to}}$ is the transient outward $\mathrm{K}^{+}$current, $I_{\mathrm{Kur}}, I_{\mathrm{Kr}}$, and $I_{\mathrm{Ks}}$ are the ultrarapid, rapid, and slow rectifier currents. The quantity $I_{\mathrm{Ca}}=I_{\mathrm{Ca}, \mathrm{L}}$ is the L-type $\mathrm{Ca}^{2+}$ current, while $I_{\mathrm{b}}$ is the the background current for sodium $\mathrm{Na}^{+}$and calcium $\mathrm{Ca}^{2+}$

$$
I_{\mathrm{b}}=I_{\mathrm{b}, \mathrm{Na}}+I_{\mathrm{b}, \mathrm{Ca}}
$$

Finally, $I_{\mathrm{p}}$ collects the actions of pumps and ion exchangers, designed to put back into balance the ion concentrations at rest:

$$
I_{\mathrm{p}}=I_{\mathrm{NaCa}}+I_{\mathrm{NaK}}+I_{\mathrm{p}, \mathrm{Ca}},
$$

where $I_{\mathrm{NaCa}}$ is the sodium-calcium pump, $I_{\mathrm{NaK}}$ is the sodium-potassium pump, and $I_{\mathrm{p}, \mathrm{Ca}}$ is the calcium exchanger.

The model handles the intracellular concentrations $\left[\mathrm{Na}^{+}\right]_{i},\left[\mathrm{~K}^{+}\right]_{i}$, and $\left[\mathrm{Ca}^{2+}\right]_{i}$. The intracellular calcium buffering by the sarcoplasmic reticulum system (SR) is described by the calcium concentrations in the uptake (NSR), and release (JSR) compartments of the sarcoplasmic reticulum, denoted by $\left[\mathrm{Ca}^{2+}\right]_{\text {up }}$ and $\left[\mathrm{Ca}^{2+}\right]_{\text {rel }}$ respectively.

In the model, no extracellular cleft space is included, the membrane capacitance is $c_{m}=100 \mathrm{pF}$, the lenght and diameter of the cells are set to 100 and $16 \mu \mathrm{m}$, respectively, and the cell compartment volumes are the same ones used in the phase-II Luo-Rudy model (LR2, [35]). Denoting by $E_{X}$ the equilibrium potential for ion $X$, and with $g_{X}$ its maximal conductance, from Nerst equation, $E_{X}$ is given by

$$
E_{X}=\frac{R T}{z F} \log \frac{[X]_{e}}{[X]_{i}},
$$

where $R$ is the gas constant, $T$ is the absolute temperature, $F$ is the Faraday constant, $z=1$ for $\mathrm{Na}^{+}$and $\mathrm{K}^{+}, z=2$ for $\mathrm{Ca}^{2+}$, and $[X]_{e}$ and $[X]_{i}$ denote the external and 
internal concentration of ion $X$.
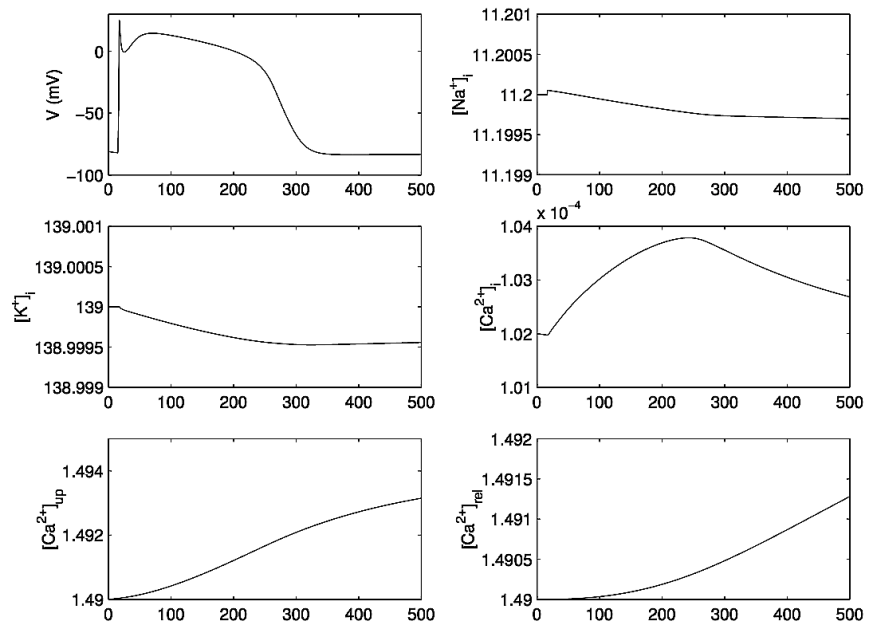

Fig. 2 CRN model: time evolution of the membrane potential and the concentration variables.

The dynamics of the concentration variables is governed by the following equations

$$
\begin{gathered}
\frac{d\left[\mathrm{Na}^{+}\right]_{i}}{d t}=\frac{-3 I_{\mathrm{NaK}}-3 I_{\mathrm{NaCa}}-I_{\mathrm{b}, \mathrm{Na}}-I_{\mathrm{Na}}}{F V_{i}} \\
\frac{d\left[\mathrm{~K}^{+}\right]_{i}}{d t}=\frac{2 I_{\mathrm{NaK}}-I_{\mathrm{K} 1}-I_{\mathrm{to}}-I_{\mathrm{Kur}}-I_{\mathrm{Kr}}-I_{\mathrm{Ks}}}{F V_{i}} \\
\frac{d\left[\mathrm{Ca}^{2+}\right]_{i}}{d t}=\left[\frac{2 I_{\mathrm{NaCa}}-I_{\mathrm{p}, \mathrm{Ca}}-I_{\mathrm{Ca}, \mathrm{L}}-I_{\mathrm{b}, \mathrm{Ca}}}{2 F V_{i}}+\frac{V_{\mathrm{up}}\left(I_{\mathrm{up}, \text { leak }}-I_{\mathrm{up}}\right)+I_{\mathrm{rel}} V_{\mathrm{rel}}}{V_{i}}\right] \times \\
\times\left[1+\frac{\alpha_{i} \beta_{i}}{\left(\left[\mathrm{Ca}^{2+}\right]_{i}+\beta_{i}\right)^{2}}+\frac{\gamma_{i} \delta_{i}}{\left(\left[\mathrm{Ca}^{2+}\right]_{i}+\delta_{i}\right)^{2}}\right]^{-1} \\
\frac{d\left[\mathrm{Ca}^{2+}\right]_{\mathrm{up}}}{d t}=I_{\mathrm{up}}-I_{\mathrm{up}, \text { leak }}-I_{\mathrm{tr}} \frac{V_{\mathrm{rel}}}{V_{\mathrm{up}}} \\
\frac{d\left[\mathrm{Ca}^{2+}\right]_{\mathrm{rel}}}{d t}=\left(I_{\mathrm{tr}}-I_{\mathrm{rel}}\right)\left[1+\frac{\alpha_{\mathrm{rel}} \beta_{\mathrm{rel}}}{\left(\left[\mathrm{Ca}^{2+}\right]_{\mathrm{rel}}+\beta_{\mathrm{rel}}\right)^{2}}\right]^{-1},
\end{gathered}
$$

where $V_{i}$ is the intracellular volume, $V_{\text {up }}$ and $V_{\text {rel }}$ are the volumes of the uptake (NSR) and release (JSR) compartments of the sarcoplasmic reticulum, $\alpha_{i}, \gamma_{i}$, and $\alpha_{\text {rel }}$ depend on the total concentrations of troponin and calmodulin in myoplasm, and of calsequestrin in JSR, while $\beta_{i}, \delta_{i}$, and $\beta_{\text {rel }}$ depend on their half saturation constants, respectively. All these three proteins are responsible of the contraction of the cell. 
In (8) and (9), $I_{\text {up,leak }}$ is the $\mathrm{Ca}^{2+}$ leak current by the JSR, $I_{\text {up }}$ is the $\mathrm{Ca}^{2+}$ uptake current by the JSR, while $I_{\text {rel }}$ is the $\mathrm{Ca}^{2+}$ release current from the JSR. Finally, in (9) and (10), $I_{\text {tr }}$ is the transfer current from NSR to JSR.

The model consists globally of 5 concentration variables and 15 gating variables. In Figure 2 and 3 we plot the time evolution of the potential and of the gating and concentration variables. For a more detailed description of the model we refer the interested reader to the original paper by Courtemanche et al. [18]. Among other popular human atrial models, we recall the ones proposed by Earm and Noble in [19], and the one proposed by Nygren and his collaborators in [40]
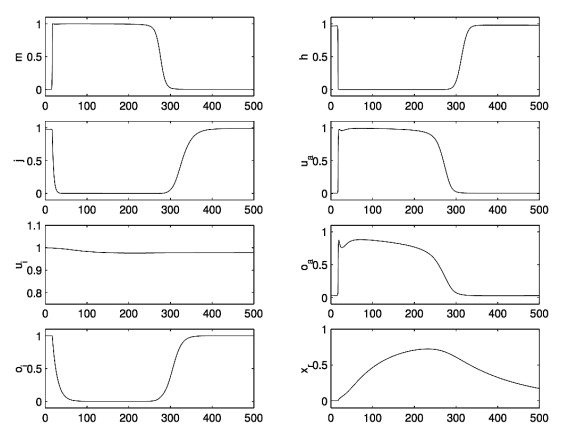
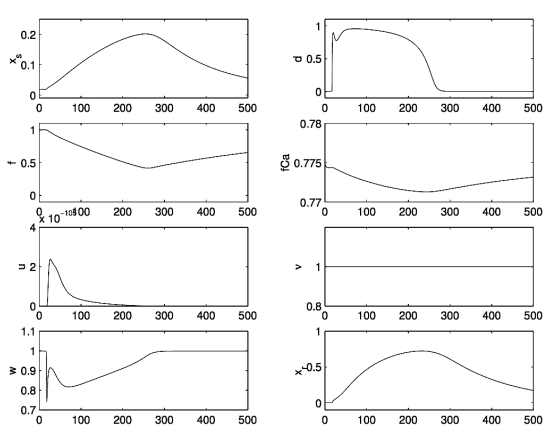

Fig. 3 CRN model: time evolution of the gating variables.

\subsection{The Luo-Rudy 1 ventricular cell model}

The Luo-Rudy Phase 1 model is among the most popular ionic model used in literature to model ventricular myocites. It consists of 6 ionic currents, 7 gating variables, and one concentration variable for the intracellular calcium, whose dynamics plays a pivotal role in the heart contraction. The total current is given by

$$
I_{i o n}=I_{N a}+I_{s i}+I_{K}+I_{K_{1}}+I_{K p}+I_{b}
$$

where the ionic currents are given by

$$
\begin{aligned}
I_{N a} & =g_{N a} m^{3} h j\left(u-E_{N a}\right) & I_{s i} & =g_{s i} d f\left(u-E_{s i}\right) \\
I_{K} & =g_{K}\left([K]_{e}\right) \xi \xi_{i}\left(u-E_{K}\right) & I_{K_{1}} & =f_{K_{1}}\left([K]_{e}, u\right)\left(u-E_{K 1}\right) \\
I_{K p} & =f_{K_{p}}(u) K_{p}\left(u-E_{K p}\right) & I_{b} & =b_{1}\left(u+b_{2}\right) .
\end{aligned}
$$

The calcium concentration satisfies the differential equation 


$$
\frac{d[\mathrm{Ca}]_{i}}{d t}=-c_{3} I_{s i}+c_{2}\left(c_{3}-[\mathrm{Ca}]_{i}\right),
$$

while the gating variables are described, within the $\mathrm{HH}$ formalism, as

$$
\frac{d w}{d t}=\alpha_{w}(1-w)-\beta_{w} w, \quad \text { with } w \in\left\{m, h, j, d, f, \xi, \xi_{i}\right\} .
$$

In (12)-(14), $I_{K_{p}}$ is the plateau current, $I_{b}$ is the background current, $f_{K_{1}}$ and $f_{K_{p}}$ are rational exponentials of the membrane potential, $g_{K}\left([K]_{e}\right)$ is a function of the extracellular potassium concentration $[K]_{e}, E_{s i}$ is linearly dependent on the natural logarithm of the intracellular calcium concentration $[\mathrm{Ca}]_{i}$, while $g_{\mathrm{Na}}, g_{s i}, b_{1}, b_{2}, c_{2}$, and $c_{3}$ are constants determined by fitting with experimental data. In the LR1 model, $\alpha_{h}, \beta_{h}, \alpha_{j}, \beta_{j}, \xi$, and $\xi_{i}$ depend on the membrane potential $u$ through functions that show different behavior with respect to a threshold. In Figure 4 we plot the temporal dynamics of the membrane potential, the calcium concentration, and the gating variables for the LR1 model.
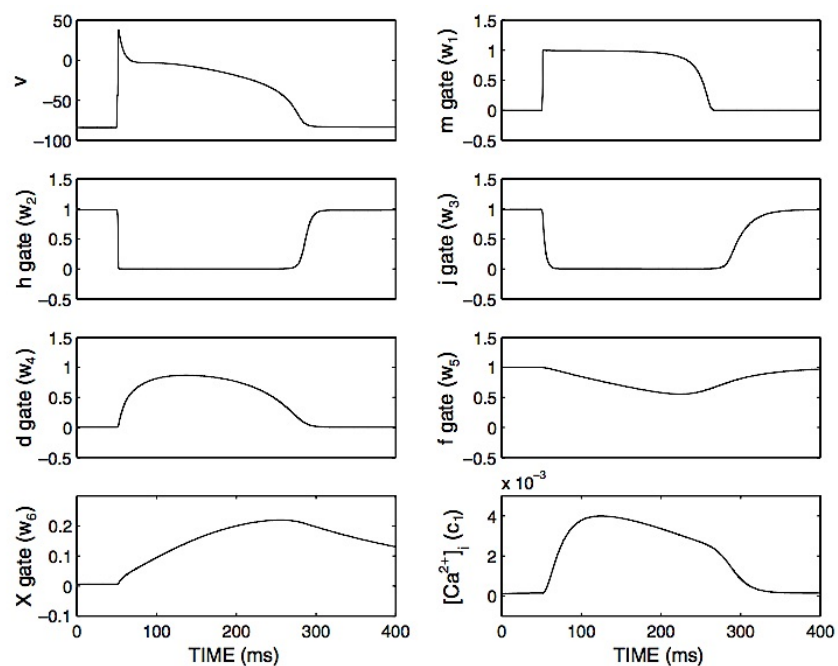

Fig. 4 Time evolution of the membrane potential, the gating variables and the $[\mathrm{Ca}]_{i}$ concentration in the LR1 model.

The LR1 model was extended, in 1994, to the Luo-Rudy phase 2 model, that includes 6 ionic currents, and 5 ionic concentrations, which allow (as in the CRN model for atrial cells) the calcium handling by the sarcoplasmic reticulum in the interior of the cell. The LR2 model was further extended by Winslow in 1999, by including experimental data from a canine heart and a more detailed modeling of the calcium dynamics, to a model featuring 25 ionic currents and 6 ionic concentrations. 


\section{The macroscopic Bidomain model for electrophysiological propagation}

The Bidomain model is commonly considered one of the most complete and accurate models to describe the propagation of the electrical potential in the myocardium tissue (see e.g. [52], [50], [27]). Such model has been derived, by an homogenization technique, starting from a periodic assembling of elongated cells surrounded by extracellular space and connected by end-to-end or side-to-side junctions (for the mathematical details we refer to $[32,14])$. The mathematical problem in naturally set in a bounded region $\Omega \subset \mathbf{R}^{3}$, which represents a portion of the heart tissue.

\subsection{Tissue and conductivities modeling}

The Bidomain model relies on representing the cardiac tissue as the superposition of two media which are both continuous and anisotropic. The intra-cellular and the extra-cellular media coexist at each point $\mathbf{x} \in \Omega$ and are separated by a cell membrane. In a natural manner, the intracellular and extracellular potential are denoted by $u_{i}$ and $u_{e}$, respectively, while their difference $u=u_{i}-u_{e}$ expresses the membrane potential.

The conductivity of the cardiac cells depends upon their orientation, featuring preferential pathway along gap junctions (see Figure 5, left), and in the most general case the conductivity tensor is anisotropic. The structure of the cardiac cells can be modeled, following Le Grice et al. ([33]) as a sequence of muscular layers going from endocardium to epicardium (see also [56]). Anatomical studies show that the fibers direction rotates counterclockwise from epicardium to endocardium and that they are arranged in sheets, running across the myocardial wall $([4,52])$. In any point $\mathbf{x}$ it is then possible to identify an orthonormal triplet of directions, $\mathbf{a}_{l}(\mathbf{x})$ along the fiber, $\mathbf{a}_{t}(\mathbf{x})$ orthogonal to the fiber direction and in the fiber sheet and $\mathbf{a}_{n}(\mathbf{x})$ orthogonal to the sheet (see Figure 5, right, for a schematic representation). The intra and extracellular media present different conductivity values in each direction. At point $\mathbf{x} \in \Omega$, we denote by $\sigma_{\tau}^{l}(\mathbf{x}), \sigma_{\tau}^{n}(\mathbf{x})$, and $\sigma_{\tau}^{t}(\mathbf{x})$ with $\tau=i, e$ the intra and extracellular conductivities in the $\mathbf{a}_{l}(\mathbf{x}), \mathbf{a}_{t}(\mathbf{x})$ and $\mathbf{a}_{n}(\mathbf{x})$ direction, respectively.

The intra and extracellular local anisotropic conductivity tensors read, for $\tau=i, e$,

$$
\mathbf{D}_{\tau}(\mathbf{x})=\sigma_{\tau}^{l}(\mathbf{x}) \mathbf{a}_{l}(\mathbf{x}) \mathbf{a}_{l}^{T}(\mathbf{x})+\sigma_{\tau}^{t}(\mathbf{x}) \mathbf{a}_{t}(\mathbf{x}) \mathbf{a}_{t}^{T}(\mathbf{x})+\sigma_{\tau}^{n}(\mathbf{x}) \mathbf{a}_{n}(\mathbf{x}) \mathbf{a}_{n}^{T}(\mathbf{x})
$$

We assume that $\mathbf{D}_{\tau}$ fulfills in $\Omega$ a uniform elliptic condition.

A common practical hypothesis, followed by many authors (see, e.g. [13]) is axial isotropy: the myocardium tissue is assumed to feature the same conductivity in both the tangential and normal direction $\left(\sigma_{i, e}^{t}=\sigma_{i, e}^{n}\right)$. Under this hypotesis, the conductivity tensors, for $\tau=i, e$, simplify in

$$
\mathbf{D}_{\tau}(\mathbf{x})=\sigma_{\tau}^{t} \mathbf{I}+\left(\sigma_{\tau}^{l}-\sigma_{\tau}^{t}\right) \mathbf{a}_{l}(\mathbf{x}) \mathbf{a}_{l}^{T}(\mathbf{x} .
$$



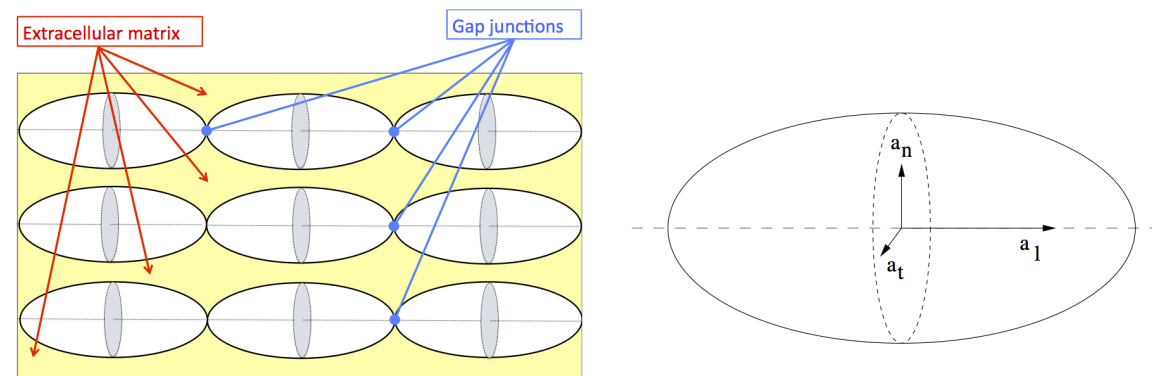

Fig. 5 Left: schematic representation of the fibers structure. Right: the cell reference frame

\subsection{Quasi-static electromagnetic field}

The propagation of an action potential across the myocardium generates, at the macroscopic level, an electrical signal that can be measured, and whose temporal dynamics is described by Maxwell's equations.

In a conducting body, the electrical current density is governed by Ohm's Law,

$$
\mathbf{J}=\mathbf{D} E,
$$

where $\mathbf{D}$ is the conductivity of the medium, and $E$ is the electrical field.

Faraday's law relates the time derivative of the magnetic field with the rotational of the electrical field:

$$
\frac{\partial B}{\partial t}+\nabla \times E=0
$$

Given the temporal scale of the AP and the spatial scale of the heart, variations of the magnetic field can be neglected, leading to the quasi-static assumption

$$
\frac{\partial B}{\partial t} \sim 0 \quad \Longrightarrow \quad \nabla \times E=0 .
$$

Since the electric field $E$ can be assumed irrotational, there exists some potential $u$ such that $E=\nabla u$, and the current in the conducting medium can then be expressed in terms of such potential, as

$$
\mathbf{J}=\mathbf{D} \nabla u
$$

\subsection{The Bidomain model}

The assumption of coexistence of intra- and extracellular media entails that in each point of the domain $\mathbf{x} \in \Omega$, two currents exist and are given by 


$$
\mathbf{J}_{i}=\mathbf{D}_{i} \nabla u_{i} \quad \text { and } \quad \mathbf{J}_{e}=\mathbf{D}_{e} \nabla u_{e},
$$

where $\mathbf{D}_{i}$ and $\mathbf{D}_{e}$ represent the conductivities of the intra- and extra-cellular medium, respectively. For any given small volume $V$, the charge conservation principle entails that the total current entering the volume must equal the total current leaving it. Within our framework, this principle amounts to balance the current flowing between the intracellular and extracellular space, as

$$
\int_{\partial V} \mathbf{n} \cdot\left(\mathbf{J}_{i}+\mathbf{J}_{e}\right) d s=0 .
$$

By a straightforward application of the divergence theorem, we have, for any given small volume $V$, that

$$
\nabla \cdot\left(\mathbf{J}_{i}+\mathbf{J}_{e}\right)=0
$$

and, from (19), the current balance is given by

$$
\nabla \cdot\left(\mathbf{D}_{i} \nabla u_{i}\right)+\nabla \cdot\left(\mathbf{D}_{i} e \nabla u_{e}\right)=0 .
$$

The current flowing from one domain to the other must equal the cell membrane current, that is given in (1). We thus obtain

$$
\nabla \cdot\left(\mathbf{D}_{i} \nabla u_{i}\right)=-\nabla \cdot\left(\mathbf{D}_{e} \nabla u_{e}\right)=\chi\left(C_{m} \frac{\partial u}{\partial t}+I_{i o n}\right)
$$

where $\chi$ is the surface to volume ratio of the cell.

Depending on the way the three terms in system (23) are grouped, two different formulation of the Bidomain model emerge.

\section{Parabolic-Parabolic formulation of the Bidomain model}

By equaling the first and second terms to the third one in (23), the Bidomain model results in a system of two nonlinear parabolic reaction-diffusion equations:

$$
\begin{array}{r}
\chi C_{m} \frac{\partial u}{\partial t}-\nabla \cdot\left(\mathbf{D}_{i} \nabla u_{i}\right)+\chi I_{i o n}=0 \\
-\chi C_{m} \frac{\partial u}{\partial t}-\nabla \cdot\left(\mathbf{D}_{e} \nabla u_{e}\right)-\chi I_{i o n}=0
\end{array}
$$

This formulation is known in literature as Parabolic-Parabolic (PP). The problem is completed by suitable initial conditions, and by homogeneous Neumann boundary conditions on $\partial \Omega$, modeling an insulated myocardium.

The complete PP formulation of the Bidomain model reads: 


$$
\begin{cases}\chi C_{m} \frac{\partial u}{\partial t}-\nabla \cdot\left(\mathbf{D}_{i} \nabla u_{i}\right)+\chi I_{\text {ion }}=I_{i}^{a p p} & \text { in } \Omega \times(0, T) \\ -\chi C_{m} \frac{\partial u}{\partial t}-\nabla \cdot\left(\mathbf{D}_{e} \nabla u_{e}\right)-\chi I_{i o n}=I_{e}^{a p p} & \text { in } \Omega \times(0, T) \\ u=u_{i}-u_{e} & \text { in } \Omega \times(0, T) \\ \frac{\partial w}{\partial t}=R(u, w) & \text { in } \Omega \times(0, T) \\ \mathbf{n}^{T} \mathbf{D}_{i} \nabla u_{i}=0, \quad \mathbf{n}^{T} \mathbf{D}_{e} \nabla u_{e}=0 & \text { on } \partial \Omega \times(0, T) \\ u_{i}(\mathbf{x}, 0)=u_{i, 0}, \quad u_{e}(\mathbf{x}, 0)=u_{e, 0}, \quad w(\mathbf{x}, 0)=w_{0} & \text { in } \Omega .\end{cases}
$$

In (25), $\mathbf{n}$ is the unit normal outward-pointing vector on the surface. As a consequence of the Gauss theorem, the applied external stimuli must fulfill the compatibility condition

$$
\int_{\Omega} I_{i}^{a p p} d \mathbf{x}=\int_{\Omega} I_{e}^{a p p} d \mathbf{x}
$$

System (24) consists of two parabolic reaction diffusion equations for $u_{i}$ and $u_{e}$ where the vector of time derivatives is multiplied by a singular matrix. The system is thus said to be degenerate. The transmembrane potential $u$ is uniquely determined, while the intra and extracellular potentials $u_{i}$ and $u_{e}$ are determined up to the same function of time, whose value is usually obtained by imposing that the extracellular potential $u_{e}$ has zero mean on $\Omega\left(\int_{\Omega} u_{e} d x=0\right)$.

The PP formulation has been commonly used by several scientists. In particular, this formulation has been particularly popular in theoretical studies for wellposedeness analysis of the problem. Little is known on degenerate reaction-diffusion systems such as the Bidomain model. We refer the reader to [14] for existence, uniqueness and regularity results, both at the continuous and the semi-discrete level, and to [53] for a convergence analysis of finite elements approximations. Both papers deal with the FitzHugh-Nagumo (FHN) model of the gating system. For wellposedeness analysis of the Bidomain problem associated with different ionic models see [2], [3], and [61].

More results are known on the related eikonal approximation describing the propagation of excitation front (see for instance $[8,9,31]$ ), and a mathematical analysis of the Bidomain model using $\Gamma$-convergence theory can be found in [1].

\section{Parabolic-Elliptic formulation of the Bidomain model}

By equaling the first term to both the second and the third one in (23), and using the fact that $u_{i}=u+u_{e}$, the Bidomain model results in a system of one nonlinear parabolic reaction-diffusion equation, and a linear elliptic equation: 


$$
\begin{array}{r}
\chi C_{m} \frac{\partial u}{\partial t}-\nabla \cdot\left(\mathbf{D}_{i} \nabla u_{i}\right)-\nabla \cdot\left(\mathbf{D}_{i} \nabla u_{e}\right)+\chi I_{i o n}=0 \\
\nabla \cdot\left(\mathbf{D}_{i} \nabla u\right)+\nabla \cdot\left(\left(\mathbf{D}_{i}+\mathbf{D}_{e}\right) \nabla u_{e}\right)=0
\end{array}
$$

This formulation is known in literature as Parabolic-Elliptic (PE). As for the PP formulation, the problem is completed by suitable initial and boundary conditions, and the complete PE formulation of the Bidomain model reads:

$$
\begin{cases}\chi C_{m} \frac{\partial u}{\partial t}-\nabla \cdot\left(\mathbf{D}_{i} \nabla u_{i}\right)+\chi I_{\text {ion }}=I_{i}^{a p p} & \text { in } \Omega \times(0, T) \\ \nabla \cdot\left(\mathbf{D}_{i} \nabla u\right)+\nabla \cdot\left(\left(\mathbf{D}_{i}+\mathbf{D}_{e}\right) \nabla u_{e}\right)=0 & \text { in } \Omega \\ \frac{\partial w}{\partial t}=R(u, w) & \text { in } \Omega \times(0, T) \\ \mathbf{n}^{T} \mathbf{D}_{i} \nabla u_{i}=0, \quad \mathbf{n}^{T} \mathbf{D}_{e} \nabla u_{e}=0 & \text { on } \partial \Omega \times(0, T) \\ u_{i}(\mathbf{x}, 0)=u_{i, 0}, \quad u_{e}(\mathbf{x}, 0)=u_{e, 0}, \quad w(\mathbf{x}, 0)=w_{0} & \text { in } \Omega .\end{cases}
$$

Differently from the PP formulation, system (27) does not consist of two parabolic equations for $u_{i}$ and $u_{e}$ where the vector of time derivatives is multiplied by a singular matrix. Nevertheless, also system (27) is degenerate, since the elliptic equation in (27) is in practice a Laplacian with homogeneous Neumann boundary conditions, whose solution is known only up to a constant. Also in this formulation, thus, the transmembrane potential $u$ is uniquely determined, while the intra and extracellular potentials $u_{i}$ and $u_{e}$ are determined up to the same function of time, whose value is again obtained by imposing zero mean to the extracellular potential on $\Omega$ $\left(\int_{\Omega} u_{e} d x=0\right)$.

By letting $\lambda_{m}=\min \left\{\sigma_{e}^{l} / \sigma_{i}^{l}, \sigma_{e}^{t} / \sigma_{i}^{t}\right\}$ and $\lambda_{M}=\max \left\{\sigma_{e}^{l} / \sigma_{i}^{l}, \sigma_{e}^{t} / \sigma_{i}^{t}\right\}$, an alternative PE formulation can be obtained by linear combinations of the equations in (24), with coefficients $\left(\frac{\lambda}{1+\lambda},-\frac{1}{1+\lambda}\right), \lambda_{m} \leq \lambda \leq \lambda_{M}$, and $(1,1)$ :

$$
\begin{aligned}
\chi C_{m} \frac{\partial u}{\partial t}-\nabla \cdot\left[\frac{\lambda \mathbf{D}_{i}}{1+\lambda} \nabla u\right]-\nabla \cdot & {\left[\frac{\lambda \mathbf{D}_{i}-\mathbf{D}_{e}}{1+\lambda} \nabla u_{e}\right]+\chi I_{\text {ion }}(u)=I^{a p p} } \\
-\nabla \cdot\left[\mathbf{D}_{i} \nabla u+\left(\mathbf{D}_{i}+\mathbf{D}_{e}\right) \nabla u_{e}\right] & =\widetilde{I}^{a p p},
\end{aligned}
$$

where we have set $I^{a p p}=\frac{\lambda I_{i}^{a p p}+I_{e}^{a p p}}{1+\lambda}$ and $\widetilde{I}^{a p p}=I_{i}^{a p p}-I_{e}^{a p p}$.

The (PE) formulation of the Bidomain problem has been widely used for numerical simulations, in particular among the Bioengineering community, serving as the basis for the development of efficient preconditioners. 


\subsection{A simplified model: the Monodomain}

If we assume the anisotropy ratio to be the same in the two media, the Bidomain model reduces to a simpler one, called Monodomain. Its derivation can be obtained in different ways, the common underlying hypothesis being a proportionality assumption between the intracellular and the extracellular conductivity tensors, namely $\mathbf{D}_{e}=\lambda \mathbf{D}_{i}$, where $\lambda$ is a constant to be properly chosen. For instance, under assumption (16), $\lambda$ can be devised through a minimization procedure, as

$$
\lambda=\operatorname{argmin} J(\lambda), \quad J(\lambda)=\left(\frac{\sigma_{e}^{l}-\lambda \sigma_{i}^{l}}{1+\lambda}\right)^{2}+2\left(\frac{\sigma_{e}^{t}-\lambda \sigma_{i}^{t}}{1+\lambda}\right)^{2}
$$

for given values of the conductivities. A time dependent choice of the parameter $\lambda$ has been proposed in [39].

After defining $\mathbf{D}:=\mathbf{D}_{i}+\mathbf{D}_{e}$ and $\mathbf{D}_{M}:=\mathbf{D}_{e} \mathbf{D}^{-1} \mathbf{D}_{i}$, the first equation in (29) can be rearranged as

$\chi C_{m} \frac{\partial u}{\partial t}-\nabla \cdot \mathbf{D}_{M} \nabla u+\nabla \cdot\left[\left(\mathbf{D}_{e} \mathbf{D}^{-1}-\frac{\lambda}{1+\lambda} \mathbf{I}\right)\left(\mathbf{D}_{i} \nabla u+\mathbf{D} \nabla u_{e}\right)\right]+\chi I_{i o n}(u, \mathbf{w})=I^{a p p}$

and, since the proportionality assumption $\mathbf{D}_{e}=\lambda \mathbf{D}_{i}$ entails $\mathbf{D}_{e} \mathbf{D}^{-1}-\frac{\lambda}{1+\lambda} \mathbf{I}=\mathbf{0}$, a formulation of the Monodomain model (see $[6,30])$ is then obtained from (30) as

$$
\chi C_{m} \frac{\partial u}{\partial t}-\nabla \cdot \mathbf{D}_{M} \nabla u+\chi I_{i o n}(u, \mathbf{w})=I^{a p p}
$$

The problem is completed by suitable initial conditions, and by homogeneous Neumann boundary conditions on $\partial \Omega$, and reads:

$$
\begin{cases}c_{m} \frac{\partial u}{\partial t}-\operatorname{div}\left(\mathbf{D}_{M} \nabla u\right)+I_{i o n}(u, w)=I^{a p p} & \text { in } \Omega \times(0, T) \\ \frac{\partial w}{\partial t}-R(u, w)=0 & \text { in } \Omega \times(0, T) \\ \mathbf{n}^{T} D \nabla u=0 & \text { in } \partial \Omega \times(0, T) \\ u(\mathbf{x}, 0)=u_{0}(\mathbf{x}), \quad w(\mathbf{x}, 0)=w_{0}(\mathbf{x}), & \text { in } \Omega,\end{cases}
$$

Such model consists of a single parabolic reaction-diffusion equation for the transmembrane potential $u$ coupled with and ODE system for the gating and concentration variables. Differently from the Bidomain, several theoretical results on reaction-diffusion equations can be applied to the Monodomain model, which features a unique solution $(u, w)$, resulting in a much easier to solve problem after numerical discretization. In many applications the Monodomain model is accurate enough to capture the desired dynamics and effects of the action potential propagation. In [47], Potse and his collaborators compare the action potential propagation 
velocities using Monodomain and Bidomain, observing that the Monodomain solution propagates a bit slower (2\%) than the Bidomain one, and conclude that "in absence of applied currents, propagating of action potentials on the scale of a human heart can be studied with a Monodomain model". However, the Bidomain model becomes necessary when current stimuli are applied in the extracellular space. As a consequence, the Monodomain has been long considered inadequate to simulate defibrillation [59]. In recent works by Y. Coudiere and his collaborators, [16, 17], a proper generalisation of the boundary conditions has been introduced, that allows an external stimuli to be applied directly at the Monodomain model.

In Figure 6 we plot the difference in the propagation between Bidomain and Monodomain on a slab with the principal fibers axis oriented horizontally from left to right. The difference in the propagation speed between the two models can be clearly appreciated. In addition, it is evident that the Monodomain error, although globally significant, is minimal in both directions along and across the fibers. An accurate knowledge of the fibers arrangements would strongly reduce the error when the Monodomain model is used for patient-specific simulation. Still, such arrangement, although having common features, is highly individual, and, unfortunately, a definitive knowledge of the fibers distribution is not available yet. Modern advanced medical imaging techniques, such as DTI (Diffusion Tensor Imaging) allow an accurate mapping of the fibers direction, making the Monodomain a viable and cheaper alternative to heavy Bidomain simulations.
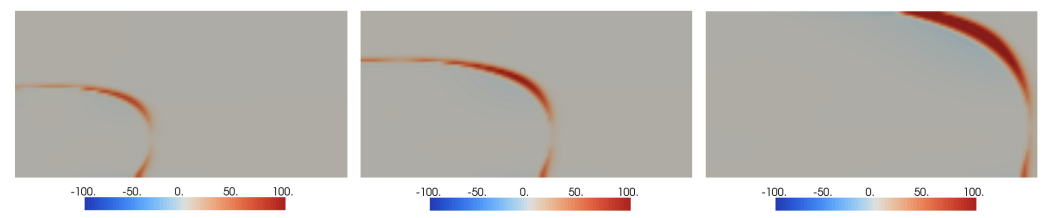

Fig. 6 Difference in the propagation of the membrane potential between Bidomain and Monodomain simulation with fibers oriented along the $x$ axis (from [26]).

\section{Numerical approximation}

We give here a brief introduction to the numerical approximation of the models presented in the previous Section. In what follows we do not rely on a specific choice for the ionic model describing the cell membrane currents. Thus, from now on, we will simply denote by $I_{i o n}(u, w)$ the ionic current. For more detailed description, we refer the interested reader to $[10,11,63]$. 


\subsection{Time marching scheme}

For the sake of simplicity in presentation, we consider a fixed time step $\Delta t$, even if effective time adaptive scheme have been developed in the literature (see e.g. [7, 46]), and we denote with superscript $n$ the variables computed at time $t^{n}=n \Delta t$. The Bidomain and Monodomain systems can be advanced in time by an implicit-explicit (IMEX) scheme: moving from time step $t^{n}$ to $t^{n+1}$, the gating and concentration variables $w$ are updated first, and used to compute the new values for the electric potentials.

Let the ionic variables vector $w \in \mathbf{R}^{m}$ be arranged as $w=(g, c)^{T}$, where $g \in \mathbf{R}^{p}$ represent the gating variables, while $c \in \mathbf{R}^{q}$ represent the concentration variables $(p+q=m)$. Owing to the Hodgkin-Huxley formalism (3)-(4), the components of $g$ are first integrated exactly in time on $(0, \Delta t)$, upon an appropriate linearisation around the membrane potential at the previous time step

$$
g_{j}^{n+1}=g_{j \infty}\left(u^{n}\right)+\left(g_{j}^{n}-g_{j \infty}\left(u^{n}\right)\right) \exp \left(-\frac{\Delta t}{\tau_{g_{j}}\left(u^{n}\right)}\right),
$$

then the concentration variables $c$ are integrated by a backward Euler scheme, taking into account the updated values $g^{n+1}$,

$$
\frac{c^{n+1}-c^{n}}{\Delta t}=R_{c}\left(u^{n}, g^{n+1}, c^{n}\right)
$$

where $R_{c}$ are the rows in (2) associated with $c$. The time step is selected to guarantee stability to the time advancing scheme.

The electric potentials are then updated by solving on $\Omega$ a semi-implicit problem, where the linear diffusion term is discretized implicitely, while the nonlinear reaction term (the ionic current $I_{i o n}(u, w)$ ) is treated explicitely with respect to the membrane potential $u$. While taking into account the updated values of the gating and concentration variables $w^{n+1}$, this allows to skip the computationally expensive solution of nonlinear systems.

Within this framework, the semi-discrete version of the Bidomain PP formulation (24), solves for $0<n \leq N=T / \Delta t$,

$$
\left\{\begin{array}{l}
\chi C_{m} \frac{u^{n+1}-u^{n}}{\Delta t}-\nabla \cdot\left(\mathbf{D}_{i} \nabla u_{i}^{n+1}\right)=I_{i}^{a p p}-\chi I_{\text {ion }}\left(u^{n}, w^{n+1}\right) \\
-\chi C_{m} \frac{u^{n+1}-u^{n}}{\Delta t}-\nabla \cdot\left(\mathbf{D}_{e} \nabla u_{e}^{n+1}\right)=I_{e}^{a p p}+\chi I_{\text {ion }}\left(u^{n}, w^{n+1}\right) \\
u_{i}^{0}(\mathbf{x})=u_{i, 0}(\mathbf{x}) \quad u_{e}^{0}(\mathbf{x})=u_{e, 0}(\mathbf{x}) \\
\left.\mathbf{n}^{T} \mathbf{D}_{i} \nabla u^{n+1}\right|_{\partial \Omega}=\left.0 \quad \mathbf{n}^{T} \mathbf{D}_{e} \nabla u_{e}^{n+1}\right|_{\partial \Omega}=0 .
\end{array}\right.
$$

Similarly, the semi-discrete version of PE formulation, solves for (27) 


$$
\left\{\begin{array}{l}
\chi C_{m} \frac{u^{n+1}-u^{n}}{\Delta t}-\nabla \cdot\left(\mathbf{D}_{i} \nabla u^{n+1}+\mathbf{D}_{e} \nabla u_{e}^{n+1}\right)=I^{a p p}-\chi I_{i o n}\left(u^{n}, w^{n+1}\right) \\
-\nabla \cdot\left[\mathbf{D}_{i} \nabla u^{n+1}+\left(\mathbf{D}_{i}+\mathbf{D}_{e}\right) \nabla u_{e}^{n+1}\right]=\widetilde{I}^{a p p} \\
u^{0}(\mathbf{x})=u_{0}(\mathbf{x}) \quad u_{e}^{0}(\mathbf{x})=u_{e, 0}(\mathbf{x}) \\
\left.\mathbf{n}^{T} \mathbf{D}_{i}\left(\nabla u^{n+1}+\nabla u_{e}^{n+1}\right)\right|_{\partial \Omega}=\left.0 \quad \mathbf{n}^{T} \mathbf{D}_{e} \nabla u_{e}^{n+1}\right|_{\partial \Omega}=0 .
\end{array}\right.
$$

and, for (29)

$$
\left\{\begin{array}{l}
\chi C_{m} \frac{u^{n+1}-u^{n}}{\Delta t}-\nabla \cdot\left(\frac{\lambda \mathbf{D}_{\mathbf{i}}}{1+\lambda} \nabla u^{n+1}+\frac{\lambda \mathbf{D}_{\mathbf{i}}-\mathbf{D}_{\mathbf{e}}}{1+\lambda} \nabla u_{e}^{n+1}\right)=I^{a p p}-\chi I_{\text {ion }}\left(u^{n}, w^{n+1}\right) \\
-\nabla \cdot\left[\mathbf{D}_{i} \nabla u^{n+1}+\left(\mathbf{D}_{i}+\mathbf{D}_{e}\right) \nabla u_{e}^{n+1}\right]=\widetilde{I}^{a p p} \\
u^{0}(\mathbf{x})=u_{0}(\mathbf{x}) \quad u_{e}^{0}(\mathbf{x})=u_{e, 0}(\mathbf{x}) \\
\left.\mathbf{n}^{T} \mathbf{D}_{i}\left(\nabla u^{n+1}+\nabla u_{e}^{n+1}\right)\right|_{\partial \Omega}=\left.0 \quad \mathbf{n}^{T} \mathbf{D}_{e} \nabla u_{e}^{n+1}\right|_{\partial \Omega}=0 .
\end{array}\right.
$$

In a similar manner, the semi-discrete formulation of the Monodomain model updates the membrane potential by solving, at each time step:

$$
\left\{\begin{array}{l}
\chi C_{m} \frac{u^{n+1}-u^{n}}{\Delta t}-\nabla \cdot \mathbf{D}_{M} \nabla u^{n+1}=I^{a p p}-\chi I_{i o n}\left(u^{n}, w^{n+1}\right) \\
u^{0}(\mathbf{x})=u_{0}(\mathbf{x}) \\
\left.\mathbf{n}^{T} \mathbf{D}_{M} \nabla u^{n+1}\right|_{\partial \Omega}=0 .
\end{array}\right.
$$

\subsection{Spatial discretization}

The most common approach in the literature is to look for approximate solutions to the Bidomain and Monodomain models in a finite element space. The computational domain $\Omega \subset \mathbf{R}^{3}$ is discretized in space with a regular triangulation $\mathscr{T}_{h}$, namely $\Omega=\bigcup_{j=1}^{N} T_{j}$, where each $T_{j} \in \mathscr{T}_{h}$ is obtained through an invertible affine map from a reference element $E$, a simplex (namely the thetrahedron with vertices $(0,0,0)$, $(1,0,0),(0,1,0)$, and $(0,0,1))$ or the unit cube $[0,1]^{3}$. The associated finite element spaces $X_{h}$ and $Y_{h}$ (see e.g. [48] for an introduction to finite element methods) are defined as

$$
X_{h}=\left\{\varphi_{h} \in C^{0}(\Omega) \mid \varphi_{h \mid T_{j}} \in \mathbf{P}_{k}\left(T_{j}\right)\right\}, \quad Y_{h}=\left\{\varphi_{h} \in C^{0}(\Omega) \mid \varphi_{h \mid T_{j}} \in \mathbf{Q}_{k}\left(T_{j}\right)\right\},
$$


where $\mathbf{P}_{k}\left(T_{j}\right)$ is the space of polynomials of degree at most $k$ on $T_{j}$, whereas $\mathbf{Q}_{k}\left(T_{j}\right)$ is the space of polynomials of degree at most $k$ with respect to each variable on $T_{j}$. A fully discrete problem for (33) - (36) is then obtained by applying a Galerkin procedure on their variational formulations, using as finite dimensional space $V_{h}=$ $X_{h}$ or $V_{h}=Y_{h}$, and choosing a basis for $V_{h}$. Let then $\Phi=\left\{\varphi_{j}\right\}_{j=1}^{N_{h}}$ be a basis for $V_{h}$. We denote by $M$ the mass matrix, and and by $K_{\tau}(\tau=i, e, M)$ the stiffness matrices with entries

$$
M^{i j}=\sum_{p=1}^{N} \int_{T_{p}} \varphi_{i} \varphi_{j} d x, \quad K_{\tau}^{i j}=\sum_{p=1}^{N} \int_{T_{p}}\left(\nabla \varphi_{j}\right)^{T} D_{\tau}(\mathbf{x}) \nabla \varphi_{i} d x
$$

Numerical evaluation of such integrals is obtained by means of suitable quadrature rules.

\subsection{Algebraic formulation}

The unknowns of the fully discrete problem are represented by vectors $\mathbf{u}, \mathbf{u}_{i}, \mathbf{u}_{e}$, and $\mathbf{w}$, storing the nodal values of $u, u_{i}, u_{e}$, and $w$, respectively.

Advancing the potentials from time $t^{n}$ to $t^{n+1}$ amount eventually to solve, at each step, a linear system. Since the Bidomain system is degenerate, the matrix associated to its discrete formulation is singular, with a one dimensional kernel spanned by $(\mathbf{1}, \mathbf{1})^{T}$, independently from its formulation. As a consequence, the transmembrane potential $\mathbf{u}^{n+1}$ is uniquely determined, as in the continuous model, while $\mathbf{u}_{i}^{n+1}$ and $\mathbf{u}_{e}^{n+1}$ are determined up to the same additive time-dependent constant with respect to a reference potential. Such constant can be determined by imposing the condition $\mathbf{1}^{T} M \mathbf{u}_{e}^{n+1}=0$. On the other hand, the matrix associated with the full discrete version of the Monodomain system is positive definite, due to the uniform ellipticity assumption on the conductivity tensors. As a consequence, the transmembrane potential $\mathbf{u}^{n+1}$ is uniquely determined for the discrete Monodomain system.

\section{Parabolic-Parabolic Bidomain formulation}

The full discretization of the PP Bidomain system (24) reads:

$$
\begin{aligned}
& {\left[\begin{array}{cc}
\frac{\chi C_{m}}{\Delta t} M+K_{i} & -\frac{\chi C_{m}}{\Delta t} M \\
-\frac{\chi C_{m}}{\Delta t} M & \frac{\chi C_{m}}{\Delta t} M+K_{i}
\end{array}\right]\left[\begin{array}{l}
\mathbf{u}_{i} \\
\mathbf{u}_{e}
\end{array}\right]^{n+1}=} \\
& \frac{\chi C_{m}}{\Delta t}\left[\begin{array}{cc}
M & -M \\
-M & M
\end{array}\right]\left[\begin{array}{l}
\mathbf{u}_{i} \\
\mathbf{u}_{e}
\end{array}\right]^{n}+\left[\begin{array}{c}
\chi M I_{i o n}^{h}\left(\mathbf{u}^{n}, \mathbf{w}^{n+1}\right)+M I_{i}^{a p p} \\
-\chi M I_{i o n}^{h}\left(\mathbf{u}^{n}, \mathbf{w}^{n+1}\right)+M I_{e}^{a p p}
\end{array}\right]
\end{aligned}
$$

The above linear system features a symmetric positive semidefinite matrix, Due to its symmetry, the system can be solved by a Preconditioned Conjugate Gradient 
algorithm (PCG, see e.g. [51]), using as initial guess the solution at the previous time step.

Parabolic-Elliptic Bidomain formulation.

The full discretization of the PE Bidomain system (28) reads:

$$
\begin{aligned}
& {\left[\begin{array}{cc}
\frac{\chi C_{m}}{\Delta t} M+K_{i} & K_{i} \\
K_{i} & K_{i}+K_{e}
\end{array}\right]\left[\begin{array}{l}
\mathbf{u} \\
\mathbf{u}_{e}
\end{array}\right]^{n+1}=} \\
& {\left[\begin{array}{l}
\frac{\chi C_{m}}{\Delta t} M \mathbf{u}^{n}+\chi M I_{i o n}^{h}\left(\mathbf{u}^{n}, \mathbf{w}^{n+1}\right)+M I_{i}^{a p p} \\
M I_{i}^{a p p}-M I_{e}^{a p p}
\end{array}\right], }
\end{aligned}
$$

while the full discretization of the (29) reads:

$$
\begin{aligned}
& {\left[\begin{array}{cc}
\frac{\chi C_{m}}{\Delta t} M+\frac{\lambda}{1+\lambda} K_{i} & \frac{\lambda}{1+\lambda} K_{i}-\frac{1}{1+\lambda} K_{e} \\
K_{i} & K_{i}+K_{e}
\end{array}\right]\left[\begin{array}{l}
\mathbf{u} \\
\mathbf{u}_{e}
\end{array}\right]^{n+1}=} \\
& {\left[\begin{array}{l}
\frac{\chi C_{m}}{\Delta t} M \mathbf{u}^{n}+\chi M I_{i o n}^{h}\left(\mathbf{u}^{n}, \mathbf{w}^{n+1}\right)+\frac{\lambda}{1+\lambda} M I_{i}^{a p p}+\frac{1}{1+\lambda} M I_{e}^{a p p} \\
M I_{i}^{a p p}-M I_{e}^{a p p}
\end{array}\right] }
\end{aligned}
$$

While the fully discrete formulation of (28) is symmetric, and can be solved by PCG, the fully discrete formulation of (29) system results naturally in a nonsymmetric matrix at the discrete level. The resulting linear system can then be solved with an iterative method such GMRES or Bi-CGSTAB (see [51]), using as initial guess the solution at the previous time step.

Monodomain

The full discretization of the Monodomain system (32) reads:

$$
\left[\frac{\chi C_{m}}{\Delta t} M+K_{m}\right][\mathbf{u}]^{n+1}=[\mathbf{f}]^{n+1}
$$

The associated matrix is naturally symmetric, and the linear system can be solved by a PCG, using as initial guess the solution at the previous time step.

\subsubsection{Computational aspects}

The numerical solution of the Bidomain system is an expensive computational task. if, on the one hand, the IMEX approach describe above allows to avoid the costly solution of nonlinear systems, the degenerate nature of the Bidomain itself entails a very ill conditioning for the linear system associated to its full discretization. To 
cope with such computational complexity, several scientists have developed in the recent years effective preconditioning strategies to reduce the high computational costs associated to its numerical solution ([10], [43], [37], [63], [44], [45], [62], [54]). Among these works, most are based on a proper decomposition of the computational domain in order to set up parallel preconditioners, or on suitable multigrid schemes still coupled with parallel architectures. The PE formulation is a popular choice in the Bioengineering community, as it is computationally more stable and allows for decoupled approaches in the solution. In fact, performing the space discretization first, results in a Differential-Algebraic system in time. It can then be natural to decouple the differential part from the algebraic one, and use the elliptic equation of the PE formulation as a corrector step in a two-level scheme (see, e.g. [12]). An efficient serial preconditioner for the PE formulation has been proposed in $[23,22]$ stemming from a suitable extension of the Monodomain model, and resulting in a lower block-triangular preconditioner for (29). Adaptive techniques have been proposed as well, to better capture the front propagation of the electrical excitation. An efficient adaptive strategy, reshaping the computational grid according to some suitable a posteriori error estimate has been introduced for instance in [7]. However, since the problem is time dependent, such approach requires to frequently recompute the mesh and interpolate between the old and the new grid, a feature that can become a serious bottleneck when simulating reentrant waves or fibrillation. Stemming from the observation that the Monodomain provides an accurate approximation of the potential in most of the region of interest, a model adaptive strategy has been proposed as well, which aims at reducing computational costs and to maintain the accuracy by solving the Bidomain problem only in (hopefully) small, critical (in physiopathological terms) regions of the domain, while the Monodomain equation is solved in the remaining regions, where the potential propagation dynamics does not require the most sophisticated model. A first version of this approach was presented in [36], where a suitable a posteriori model estimator was introduced, and an hybrid model called Hybridomain was advocated. The latter assembles the block $(1,2)$ in $(37)$ only in correspondence with the nodes identified as Bidomain ones by the model estimator, while the second equation stays untouched, and a problem the same size of the original Bidomain model has to be solved. If the constant $\lambda$ in (29) is properly chosen, the block $(1,1)$ of $(37)$ is actually the discretization of the Monodomain model. Following this consideration, an improved version of the model adaptive strategy has been introduced in [24], where only the block $(1,1)$ of (37) is solved in the Monodomain regions. The coupling between regions was based on Optimized Schwarz Methods [26, 25], a popular technique in the field of Domain Decomposition algorithms (see e.g. [21, 58]), which relies on Robin transmission conditions on the interface between subdomains. 


\section{References}

1. L. Ambrosio, P. Colli Franzone, and G. Savaré. On the asymptotic behaviour of anisotropic energies arising in the cardiac bidomain model. Interfaces Free Bound., 2:213-266, 2000.

2. M. Bendahmane and K.H. Karlsen Analysis of a class of degenerate reaction-diffusion systems and the bidomain model of cardiac tissue Netw. Heterog. Media, 1(1), 2006:185-218.

3. Y. Bourgault, Y. Coudière and Charles Pierre Existence and uniqueness of the solution for the bidomain model used in cardiac electrophysiology Nonlinear Anal.: Real World Appl., 10(1), 2009:458-482.

4. R. H. Clayton, O. M. Bernus, E. M. Cherry, H. Dierckx, F. H. Fenton, L. Mirabella, A. V. Panfilov, F. B. Sachse, G. Seemann, H. Zhang, Models of cardiac tissue electrophysiology: Progress, challenges and open questions, Progress in biophysics and molecular biology 104, pp. 22-48, 2011.

5. R.H. Clayton and A.V. Panfilov. A guide to modelling cardiac electrical activity in anatomically detailed ventricles. Progress in Biophysics and Molecular Biology, 96(1-3):19-43, 2008.

6. J.C. Clements, J. Nenonen, P.K.J. Li, and M.Horacek. Activation dynamics in anisotropic cardiac tissue via decoupling. Ann. Biomed. Eng., 32(7):984-990, 2004.

7. P. Colli Franzone, P. Deuflhard, B. Erdmann, J. Lang, and L.F. Pavarino. Adaptivity in space and time for reaction-diffusion systems in electrocardiology. SIAM Journal on Scientific Computing, 28(3):942-962, 2006.

8. P. Colli Franzone and L. Guerri. Spread of excitation in 3-D models of the anisotropic cardiac tissue, I: Validation of the eikonal approach. Math. Biosci., 113:145-209, 1993.

9. P. Colli Franzone, L. Guerri, M. Pennacchio, and B. Taccardi. Spread of excitation in 3-D models of the anisotropic cardiac tissue, II: Effect of the fiber architecture and ventricular geometry. Math. Biosci., 147:131-171, 1998.

10. P. Colli Franzone and L.F. Pavarino. A parallel solver for reaction-diffusion systems in computational electrocardiology. Mathematical models and methods in applied sciences, 14(6):883-911, 2004.

11. P. Colli Franzone, L.F. Pavarino, G. Savaré. Computational electrocardiology: mathematical and numerical modeling, in Complex Systems in Biomedicine - A. Quarteroni, L. Formaggia, and A. Veneziani, editors. Springer, Milan, 2006.

12. P. Colli Franzone, LF. Pavarino, and S. Scacchi. Parallel Coupled and Uncoupled Multilevel Solvers for the Bidomain Model of Electrocardiology. in Domain Decomposition Methods in Science and Engineering XXI Springer International Publishing, 257-264, 2014.

13. P. Colli Franzone, L.F. Pavarino, and B. Taccardi. Simulating patterns of excitation, repolarization and action potential duration with cardiac Bidomain and Monodomain models. Math. Biosc., 197:35-66, 2005.

14. P. Colli Franzone and G. Savaré. Degenerate evolution systems modeling the cardiac electric field at micro and macroscopic level. In A. Lorenzi and B. Ruf, editors, Evolution Equations, Semigroups and Functional Analysis, pages 49-78. Birkhauser, 2002.

15. S. Cortassa, M.A. Aon, E. and Marbán, R.L. Winslow, and B. O'Rourke. An integrated model of cardiac mitochondrial energy metabolism and calcium dynamics. Biophys $J$ 84, p. 2734-2755, 2003.

16. Y. Coudière, Y. Bourgault, and M. Rioux Optimal monodomain approximations of the bidomain equations used in cardiac electrophysiology. Math Models and Methods in Applied Sciences, 24(06), 1115-1140, 2014.

17. Y. Coudière, and M. Rioux Virtual electrodes mechanisms predictions with a current-lifted monodomain model. in Computing in Cardiology, 39, pp. 837-840, 2012.

18. M. Courtemanche, R.J. Ramirez, and S. Nattel. Ionic mechanisms underlying human atrial action potential properties: insights from a mathematical model. Am. J. Physiol., 275 (Heart Circ. Physiol. 44):H301-H321, 1998.

19. Y.E. Earm and D. Noble. A model of the single atrial cell: relation between calcium current and calcium release. Pro. R. Soc. London B Biol. Sci., 240:83-96, 1990. 
20. R. FitzHugh Impulses and physiological states in theoretical models of nerve membrane. Biophysical Journal 1, 445-466, 1961.

21. M.J. Gander. Optimized Schwarz methods. SIAM J. Num. Anal., 44(2), pp. 699-731, 2006.

22. L. Gerardo-Giorda, L. Mirabella, Spectral analysis of a block-triangular preconditioner for the Bidomain system in electrocardiology Electronic Transactions on Numerical Analysis, 39, pp. 186-201, 2012.

23. L. Gerardo-Giorda, L. Mirabella, F. Nobile, M. Perego, and A. Veneziani. A model-based block-triangular preconditioner for the Bidomain system in electrocardiology. J. Comp. Phys., 228, pp. 3625-3639, 2009.

24. L. Gerardo-Giorda, L. Mirabella, M. Perego, A. Veneziani, Optimized Schwarz Methods and model adaptivity in electrocardiology simulations, in Domain Decomposition Methods in Science and Engineering XXI Springer International Publishing, pp. 265- 272, 2014.

25. L. Gerardo-Giorda and M. Perego, Optimized Schwarz Methods for the Bidomain system in electrocardiology M2AN, Vol. 47 (2), pp 583-608, 2013.

26. L. Gerardo-Giorda, M. Perego, and A. Veneziani. Optimized Schwarz coupling of Bidomain and Monodomain models in electrocardiology. M2AN, Vol. 45 (2), pp. 309-334, 2011.

27. C.S. Henriquez. Simulating the electrical behavior of cardiac tissue using the bidomain model. Crit. Rev. Biomed. Engrg., 21:1-77, 1993.

28. A.L. Hodgkin and A.F. Huxley. A quantitative description of membrane current and its application to conduction and excitation in nerve. J. Physiol., 117:500-544, 1952.

29. V. Iyer, R. Mazhari, and R. Winslow A Computational Model of the Human Left-Ventricular Epicardial Myocyte. Biophys J 87, p. 1507-1525, 2004

30. J.P. Keener. Direct activation and defibrillation of cardiac tissue. J. Theor. Biol., 178:313-324, 1996.

31. J.P. Keener. An eikonal-curvature equation for the action potential propagation in myocardium. J. Math. Biol., 29:629-651, 1991.

32. J.P. Keener and J. Sneyd. Mathematical Physiology. Springer-Verlag, 1998.

33. J. Le Grice, B.H. Smaill, and P.J. Hunter. Laminar structure of the heart: a mathematical model. Am. J. Physiol., 272 (Heart Circ. Physiol.)(41):H2466-H2476, 1995.

34. G.T. Lines, M.L. Buist, P. Grottum, A.J. Pullan, J. Sundnes, and A. Tveito. Mathematical models and numerical methods for the forward problem in cardiac electrophysiology Comput. Visual. Sci,5:215-239, 2003.

35. C. Luo and Y. Rudy. A dynamic model of the cardiac ventricular action potential. Circ. Res., 74:1071-1096, 1994.

36. L. Mirabella, F. Nobile, and A. Veneziani. An a posteriori error estimator for model adaptivity in electrocardiology. Comp. Meth. Appl. Mech. Engrg., Vol 200 (37-40), pp. 2727-2737, 2011.

37. M. Munteanu, L. F. Pavarino, and S. Scacchi. A scalable Newton-Krylov-Schwarz method for the Bidomain reaction-diffusion system. SIAM Journal on Scientific Computing, 31(5):3861-3883, 2009.

38. JC Neu, and W. Krassowska Homogenization of syncytial tissues. Crit. Rev. Biomed. Eng. 21, p.137-199, 1993.

39. B. F. Nielsen, T. S. Ruud, G. T. Lines, and A. Tveito. Optimal monodomain approximation of the bidomain equations. Appl. Math. Comp., 184:276-290, 2007.

40. A. Nygren, C. Fiset, L.Firek, J.W. Clark, D.S. Lindblad, R.B. Clark, and W.R Giles. Mathematical model of an adult human atrial cell: the role of $\mathrm{K}+$ currents in repolarization. Circ. Res., 82:63-81, 1998.

41. T. O'Hara, L. Virág, A Varró, and Y. Rudy Simulation of the undiseased human cardiac ventricular action potential: model formulation and experimental validation. PLoS Comput Biol 7, 2011.

42. A. Pashaei, D. Romero, R. Sebastian, O. Camara, A.F. Frangi. Fast Multiscale Modeling of Cardiac Electrophysiology Including Purkinje System. IEEE Trans. on Biomed. Engrng., 58(10), 2956-2960, 2011

43. L. F. Pavarino and S. Scacchi. Multilevel additive Schwarz preconditioners for the Bidomain reaction-diffusion system. SIAM J. Sci. Comp., 31(1):420-443, 2008. 
44. M. Pennacchio and V. Simoncini. Efficient algebraic solution of rection-diffusion systems for the cardiac excitation process. J. Comput. Appl. Math., 145(1):49-70, 2002.

45. M. Pennacchio and V. Simoncini. Algebraic multigrid preconditioners for the bidomain reactiondiffusion system. Appl. Num. Math., 59(12):3033-3050, 2009.

46. M. Perego and A. Veneziani. An efficient generalization of the Rush-Larsen method for solving electro-physiology membrane equations. Electronic Transaction on Numerical Analisys, 35:234-256, 2009.

47. M. Potse, B. Dubé, J. Richer, and A. Vinet. A comparison of Monodomain and Bidomain Reaction-Diffusion models for Action Potential Propagation in the Human Heart. IEEE Trans. Biomed. Eng., 53(12):2425-2435, 2006.

48. A. Quarteroni and A. Valli. Numerical Approximation of Partial Differential Equations. Springer-Verlag, Berlin, 1994.

49. J. Rogers and A. McCulloch. A collocation-Galerkin finite element model of cardiac action potential propagation. IEEE Transactions on Biomedical Engineering, 41:743-757, 1994.

50. B.J. Roth. Action potential propagation in a thick strand of cardiac muscle. Circ. Res., 68:162-173, 1991.

51. Y. Saad. Iterative Methods for Sparse Linear Systems (2nd Edition) Society for Industrial and Applied Mathematics, 2003.

52. F. B. Sachse. Computational Cardiology. Springer, Berlin, 2004.

53. S. Sanfelici. Convergence of the Galerkin approximation of a degenerate evolution problem in electrocardiology. Numer. Meth. Part. Diff. Eq., 18(2):218-240, 2002.

54. S. Scacchi. A hybrid multilevel Schwarz method for the bidomain model. Computer Methods in Applied Mechanics and Engineering, 197(45-48):4051-4061, August 2008.

55. N.G. Sepulveda, B.J. Roth, and J.P. Wikswo. Current injection into a two- dimensional anisotropic bidomain. Biophys J 55, p. 987-999, 1989.

56. D. Streeter. Gross morphology and fiber geometry in the heart. In R.M. Berne, editor, Handbook of Physiology, volume 1 (Sec. 2), pages 61-112. Williams and Wilnkins, 1979.

57. K. H. Ten Tusscher, and D. Noble, and P.J. Noble, and A.V. Panfilov. A model for human ventricular tissue. Am. J. Physiol. Heart. Circ. Physiol 286, 2004.

58. A. Toselli and O. Widlund. Domain Decomposition Methods (1st edition) Springer, 2004

59. N. Trayanova. Defibrillation of the heart: insights into mechanisms from modelling studies. Experimental Physiology, 91: 323-337, 2006.

60. M. Vazquez, R. Aris, G. Houzeaux, R. Aubry, and P. Villar. A massively parallel computational electrophysiology model of the heart. International Journal for Numerical Methods in Biomedical Engineering, Vol 27(12): 1911- 1929, 2011.

61. M. Veneroni. Reaction-diffusion systems for the macroscopic bidomain model of the cardiac electric field. Nonlinear Analysis: Real World Applications, 10(2):849-868, 2009.

62. E.J. Vigmond, F. Aguel, and N.A. Trayanova. Computational techniques for solving the bidomain equations in three dimensions. IEEE Trans. Biomed. Eng., 49(11):1260-1269, 2002.

63. E.J. Vigmond, R. Weber dos Santos, A.J. Prassl, M. Deo, and G. Plank. Solvers for the caridac bidomain equations. Progress in Biophysics and Molecular Biology, 96(1-3):3-18, 2008.

64. R. Weber dos Santos, G. Planck, S. Bauer, and E.J. Vigmond. Parallel multigrid preconditioner for the cardiac bidomain model. IEEE Trans. Biomed. Eng., 51(11):1960-1968, 2004.

65. Winfree, A. T.. Varieties of spiral wave behavior: An experimentalist's approach to the theory of excitable media. Chaos 1, p. 303-334, 1991. 